\title{
The "battle" against paroxysmal atrial fibrillation: is there a role for left atrial mechanical dyssynchrony evaluated by speckle tracking?
}

\author{
Luigi Di Biase • Pasquale Santangeli • \\ J. David Burkhardt • Andrea Natale
}

Received: 13 October 2013 / Accepted: 18 October 2013 /Published online: 10 December 2013

(C) Springer Science+Business Media New York 2013

\begin{abstract}
Although several studies have shown that in patients with paroxysmal AF (PAF), pulmonary vein isolation (PVI) alone is sufficient to achieve freedom from $\mathrm{AF}[1,2]$, in some patients, success rate is not satisfactory even after multiple procedure [3].

Several studies sought to assess predictors of AF ablation failure in the setting of PAF [3, 4].

Although left atrial (LA) size, female gender, obesity, presence of scar detected by low amplitude electrograms, voltage mapping, and MRI have been proposed as a predictor of AF ablation failure, several considerations arise [3, 4].

Unpublished data from our group and from others have shown that around $20 / 30 \%$ of paroxysmal patients fail ablation procedures if these are limited to pulmonary vein isolation.
\end{abstract}

L. Di Biase $\cdot$ P. Santangeli $\cdot$ J. D. Burkhardt $\cdot$ A. Natale

Texas Cardiac Arrhythmia Institute, St. David's Medical Center,

Austin, TX, USA

L. Di Biase $(\bowtie)$

Albert Einstein College of Medicine at Montefiore Hospital,

New York, USA

e-mail: dibbia@gmail.com

L. Di Biase - A. Natale

Department of Biomedical Engineering, University of Texas, Austin, TX, USA

L. Di Biase $\cdot$ P. Santangeli

Department of Cardiology, University of Foggia, Foggia, Italy

P. Santangeli

Department of Electrophysiology, University of Pennsylvania, Philadelphia, USA

A. Natale

EP Services, California Pacific Medical Center, San Francisco, CA, USA
The absence of durable PVI is considered the major cause of recurrences, but in many cases, recurrences occur in the presence of isolated pulmonary veins (PVs) [3, 5-8].

In these patients, ablation of non-PV triggers in addition to PVI is essential to achieve success at the long-term follow-up $[1,2]$.

Several methods to disclose non-PV triggers have been proposed [9].

We believe that high dosage of isoproterenol represent the best way to disclose non-PV triggers. Common areas of nonPV triggers in the settings of paroxysmal patients are represented by the posterior wall, the roof, the anterior part of the left septum, and less frequently, the coronary sinus and in the left atrial appendage [9].

In this issue of the journal, Loghin et al. [10] sought to determine whether LA mechanical dyssynchrony as evaluated by speckle tracking echocardiography predicts PAF ablation outcomes. The authors should be commended for their effort to improve outcomes by identifying patients at higher risk for ablation failure. Importantly, the authors reported that besides LA dyssynchrony, no other echocardiographic parameters including the left atrial dimensions were identified as a predictor. This is odd with previous reports [3].

On the other hand, we should take into account that the study design could be biased by its retrospective nature, by the absence of randomization, and by the small sample size.

As reported by the authors in the limitation section of the manuscript, this study is important for the purpose of proving a concept and generating hypothesis.

Unfortunately, although challenge with isoproterenol was performed in all patients, those with triggers outside the PVs were excluded from the study. The exclusion of those patients might explain why left atrial size and other common predictor of procedural failure did not show a statistical significance [9]. 
It is possible that dissynchrony reflects a higher chance of non-PV triggers and could be used to identify a subset of patients that need ablation beyond the PVs.

In this sense, assessment of atrial dissynchrony could further improve our "battle against PAF."

\section{References}

1. Li, W. J., Bai, Y. Y., Zhang, H. Y., Tang, R. B., Miao, C. L., Sang, C. H., et al. (2011). Additional ablation of complex fractionated atrial electrograms after pulmonary vein isolation in patients with atrial fibrillation: a meta-analysis. Circulation Arrhythmia and Electrophysiology, 4, 143-148.

2. Hayward, R. M., Upadhyay, G. A., Mela, T., Ellinor, P. T., Barrett, C. D., Heist, E. K., et al. (2011). Pulmonary vein isolation with complex fractionated atrial electrogram ablation for paroxysmal and nonparoxysmal atrial fibrillation: a meta-analysis. Heart Rhythm, 8 , 994-1000.

3. Bhargava, M., Di Biase, L., Mohanty, P., et al. (2009). Impact of type of atrial fibrillation and repeat catheter ablation on long-term freedom from atrial fibrillation: results from a multicenter study. Heart Rhythm, 6, 1403-1412.

4. D'Ascenzo, F., Corleto, A., Biondi-Zoccai, G., Anselmino, M., Ferraris, F., di Biase L., et al. (2013). Which are the most reliable predictors of recurrence of atrial fibrillation after transcatheter ablation?: a meta-analysis. International Journal of Cardiology, 167, 1984-1989.

5. Lemola, K., Hall, B., Cheung, P., Good, E., Han, J., Tamirisa, K., et al. (2004). Mechanisms of recurrent atrial fibrillation after pulmonary vein isolation by segmental ostial ablation. Heart Rhythm, 1, 197-202.

6. Callans, D. J., Gerstenfeld, E. P., Dixit, S., Zado, E., Vanderhoff, M., Ren, J. F., et al. (2004). Efficacy of repeat pulmonary vein isolation procedures in patients with recurrent atrial fibrillation. Journal of Cardiovascular Electrophysiology, $15,1050-1055$.

7. Ouyang, F., Antz, M., Ernst, S., Hachiya, H., Mavrakis, H., Deger, F. T., et al. (2005). Recovered pulmonary vein conduction as a dominant factor for recurrent atrial tachyarrhythmias after complete circular isolation of the pulmonary veins: lessons from double Lasso technique. Circulation, 111, 127-135.

8. Weerasooriya, R., Khairy, P., Litalien, J., Macle, L., Hocini, M., Sacher, F., et al. (2011). Catheter ablation for atrial fibrillation: are results maintained at 5 years of follow-up? Journal of the American College of Cardiology, 57, 160166.

9. Elayi, C. S., Di Biase, L., Bai, R., Burkhardt, J. D., Mohanty, P., Santangeli, P., Sanchez, J., Hongo, R., Gallinghouse, G. J., Horton, R., Bailey, S., Beheiry, S., \& Natale, A. (2013). Administration of isoproterenol and adenosine to guide supplemental ablation after pulmonary vein antrum isolation. $J$ Cardiovasc Electrophysiol 24(11), 1199-206. doi:10.1111/jce.12252.

10. Loghin et al. (2013) in press (this editorial is attached to this paper JICE2414R1). 